\title{
Se former et se transformer : perspective critique et formation universitaire aux métiers de la formation
}

Jérôme Eneau, Eric Bertrand et Geneviève Lameul

\section{(2) OpenEdition Journals}

Édition électronique

URL : http://journals.openedition.org/ripes/585

DOI : 10.4000/ripes.585

ISSN : 2076-8427

Éditeur

Association internationale de pédagogie universitaire

Référence électronique

Jérôme Eneau, Eric Bertrand et Geneviève Lameul, « Se former et se transformer : perspective critique et formation universitaire aux métiers de la formation », Revue internationale de pédagogie de

l'enseignement supérieur [En ligne], 28(1) | 2012, mis en ligne le 20 avril 2012, consulté le 07 septembre 2020. URL : http://journals.openedition.org/ripes/585 ; DOI : https://doi.org/10.4000/ripes.585

Ce document a été généré automatiquement le 7 septembre 2020

Article L.111-1 du Code de la propriété intellectuelle. 


\section{Se former et se transformer : perspective critique et formation universitaire aux métiers de la formation}

Jérôme Eneau, Eric Bertrand et Geneviève Lameul

\section{Introduction}

1 Dans « l'ingénieuse » ingénierie de formation que Pineau (2010) appelle de ses vœux, la créativité nécessaire, en terme de dispositifs, interroge les responsables de formation dans leurs réponses à un contexte fortement contraint et à des attentes institutionnelles de plus en plus normées. Parmi les défis posés à l'université, en particulier, il s'agit d'imaginer des articulations entre savoirs pratiques issus du monde du travail et savoirs théoriques issus du monde de la recherche, dans des dispositifs qui permettent de concilier de nombreuses tensions paradoxales: donner aux professionnels qui retournent aux études les outils d'analyse de leur propre positionnement, entre prescription et émancipation; donner à ces apprenants adultes des clés de développement personnel et professionnel pour leur construction identitaire; donner enfin aux étudiants en voie de professionnalisation les grilles de lecture du monde auxquelles ils prépareront à leur tour d'autres apprenants adultes qu'eux-mêmes, entre acquisition de compétences, réflexivité vis-à-vis de leur propres pratiques et construction d'une démarche éthique.

2 Notre contribution à la production de savoirs sur cet « archipel» de l'ingénierie de la formation, en cours de recomposition (Brémaud \& Guillaumin, 2010), prend ainsi la forme d'une étude de cas, à travers la description d'un dispositif mis en œuvre dans un master professionnel préparant aux métiers de la formation. Dans ce dispositif, l'alternance, la co-formation et l'accompagnement vers l'auto-formation visent tant à former des professionnels capables d'agir avec distance réflexive et jugement critique 
sur les situations de formation qu'ils doivent concevoir qu'à agir en retour sur le monde, soit en d'autres termes agir par eux-mêmes, en tant que professionnels, de manière autonome et responsable.

3 Après avoir décrit le contexte dans lequel se situe ce dispositif, nous verrons comment l'équipe pédagogique a choisi d'articuler les modes de professionnalisation au cours des deux années de ce master. Interrogeant ces modalités d'un point de vue théorique, nous présenterons alors leurs enjeux au regard de la perspective critique à laquelle l'ingénierie pédagogique fait ici référence. A travers différents exemples, nous montrerons, ensuite, les dimensions opératoires de cette ingénierie, notamment dans la place faite aux savoirs théoriques et pratiques, dans l'alternance entre périodes de stages et périodes d'analyse réflexive. Enfin, en guise d'ouverture, nous interrogerons les apports et limites de cette étude de cas qui illustre la manière dont la formation professionnelle peut tenter de construire, dans un monde contraint et aux injonctions fortes pour les professionnels, des capacités renforcées d'auto-formation, de critique et de transformation.

\section{Contexte : crise du travail et reconfiguration des activités de formation}

4 Dans le contexte d'une crise profonde et durable du travail, où chacun est enjoint de se former lui-même et de devenir l'entrepreneur de sa propre employabilité (Boltanski \& Chiapello, 1999 ; Dejours, 2010), la formation des acteurs du monde professionnel et, en particulier, des responsables de la formation et de la gestion des ressources humaines est l'enjeu de tensions croissantes. Comme dans de nombreux pays d'Europe (Leclercq \& Niclot, 2010), la formation professionnelle continue, en France, est de plus en plus orientée vers des visées sociotechniques d'adaptation du sujet à l'évolution du travail. Depuis le début des années 2000, cette finalité technicienne a été encore renforcée et la formation est plus que jamais appréhendée pour prévenir et guérir les effets d'un chômage structurel et conjoncturel.

5 Ainsi, contrairement aux objectifs humanistes qui avaient présidé à son institutionnalisation, dans les années '70 (Tremblay \& Eneau, 2006), le temps n'est plus celui du développement personnel ni de la réflexion sur la place et le rôle des ressources humaines mais celui, omniprésent, de l'efficience de la formation. Les professionnels des ressources humaines mettent désormais en avant de manière presque exclusive des objectifs tels que le retour sur investissement, la flexibilité, l'adaptabilité ou l'employabilité des collaborateurs (Eneau, 2011). Dans ce contexte, les acteurs de la formation paraissent pris au piège de logiques paradoxales avec un risque important: celui de développer et d'instituer des pratiques instrumentales au détriment d'un agir communicationnel, basé sur la réflexivité et la distance critique (Mezirow, 2001).

6 Les pratiques d'enseignement universitaire n'échappent pas à ces logiques complémentaires mais concurrentes. Tenir cette articulation est devenu, sur le plan pédagogique, un enjeu fort des formations à l'université, où traditionnellement, l'acquisition de capacités critiques et argumentatives était l'une des particularités à laquelle doit s'adjoindre désormais une injonction tout aussi forte et omniprésente de professionnalisation. Le positionnement des universités dans la préparation aux métiers de la formation, en France, est donc de plus en plus remis en cause et de 
nombreux travaux sont actuellement menés pour repenser le rôle et les apports spécifiques des universités dans la formation des professionnels de la formation (De Lescure \& Frétigné, 2010 ; Sorel \& Wittorski, 2010).

7 Dans notre université, et plus précisément au sein du master dans lequel nous occupons des responsabilités pédagogiques à Rennes ${ }^{1}$, l'enjeu a été pour nous de penser un modèle pédagogique qui puisse concilier ces paradoxes. Le modèle que nous utilisons s'appuie fortement sur la réflexivité critique ; il est développé depuis 2009 au sein d'une formation-action-recherche, nous y reviendrons, et mobilise tant les perspectives de l'apprentissage transformateur que celles de l'alternance et de l'auto-formation.

\section{Cadre théorique : former et transformer pour agir dans l'incertitude}

8 Préparer des étudiants à agir, tant comme professionnels que comme citoyens, dans le contexte actuel de crise et d'incertitude, représente une gageure pour tout responsable de formation. Si la crise du travail se double d'une crise dans le travail, dans ses finalités et dans ses conditions d'effectuation (Dejours, 2010), préparer des professionnels à agir dans ce contexte ne va pas de soi, tant pour se positionner (trouver sa propre posture), que pour défendre des priorités (morales et déontologiques) ou encore, plus globalement, trouver du sens à son action. En effet, cette crise peut être comprise, au sens le plus large, comme un processus qui touche à la fois les institutions, les organisations, les groupes et les individus. Entre dérégulation, déliaison sociale et rupture psychologique, la crise affecte les systèmes symboliques, de valeurs et de représentations; elle percute et questionne ainsi le capital expérientiel individuel et collectif, fait de schèmes de sens, de savoirs et de croyances (Bertrand, 2007). Dans ce contexte d'instabilité, il devient, dès lors, difficile, dans toutes formations universitaires initiales ou continues préparant aux métiers de la relation (éducation, soin, management, etc.), d'aider les apprenants à élaborer une posture qui puisse leur permettre de guider ultérieurement leur action (Lameul, 2009).

Comme acteurs ou futurs acteurs du monde professionnel, ces apprenants sont pris au cœur de logiques paradoxales: adapter, maitriser, rationnaliser des dispositifs, mais avec un risque important, celui d'instituer des pratiques pétries d'un agir principalement instrumental, au dépend d'un agir communicationnel plus réflexif et plus critique (Mezirow, 2000). Avec les travaux des héritiers de l'Ecole de Frankfort (Habermas, 1997, 2001 ; Honneth, 2008), nous savons pourtant que lorsque les logiques instrumentales du monde objectif sont les seules qui dirigent l'action et les apprentissages, le risque est que la formation et ses dispositifs se développent comme monde social vécu colonisé par des agencements, eux-mêmes dirigés par des logiques bancaires (Freire, 2006), empêchant alors tout agir distancié et conscientisé, tout apprentissage réellement critique.

Comment concilier ces logiques paradoxales? Ici, la perspective transformatrice de l'apprentissage proposée par Mezirow (1991, 2000, 2001 ; Mezirow \& Taylor, 2009) peut s'avérer utile pour penser la formation. Elle permet en effet d'intégrer et de confronter les expériences des apprenants adultes dans une perspective de changement à la fois individuel et collectif, soit en d'autres termes, d'articuler des dimensions autoformatrices, co-formatrices et transformatrices de cet apprentissage. Elle permet aussi, 
et peut-être surtout, de penser la formation dans des visées de changement, à la fois personnel et social (Mezirow, 2000, 2009).

11 De ce point de vue, l'utilisation des expériences vécues dans une alternance intégrative comporte un triple enjeu : il s'agit de construire, à partir de l'action, une réflexion sur sa posture professionnelle, se construire une identité ancrée dans l'expérience à la fois personnelle et collective, et construire enfin une réflexion éthique basée sur la distanciation et la mobilisation de savoirs critiques. Concrètement, la formation doit alors servir à faire émerger, voire à co-construire du sens, à partir des tensions vécues entre soi, les autres et l'environnement. Le travail sur l'expérience individuelle et collective occupe ainsi une place centrale dans le design pédagogique, en particulier lorsque l'on s'adresse à des adultes, certes forts de leurs expériences préalables, mais souvent aussi pétris de croyances et de représentations (Brookfield, 2005 ; Donaldson, 2009). Ce travail sert à prendre conscience de la façon dont les schèmes de sens sont construits par les injonctions de l'environnement, par les histoires personnelles et professionnelles et par la culture (Mezirow, 2000).

Comme démarche de construction de connaissances épistémiques, la perspective transformatrice se base sur le dialogue et l'intersubjectivité pour relater, identifier et décrire les contextes dans lesquels s'insèrent l'action, visant à déconstruire le sens normatif de ces contextes et mieux percevoir les inférences, les rôles, les logiques et surtout les modes d'interprétation présidant à l'action (Mezirow, 2000 ; Donaldson, 2009). En d'autres termes, et pour reprendre le vocabulaire de Freire, cette perspective critique en éducation des adultes se base, dans une approche dialogique et interprétative, sur différentes étapes de conscientisation, de distanciation et de recul réflexif, visant l'émancipation des apprenants par la transformation de leurs schèmes et de leurs perspectives de sens.

Enfin, cette perspective distingue, tout en les articulant, l'apprentissage instrumental et l'apprentissage communicationnel, dans le sens que leur confère Mezirow (2000) à la suite d'Habermas: logiques d'instrumentalisation (voire de manipulation de l'environnement et des autres) contre logiques de dialogue (et d'intercompréhension, incluant les sentiments, croyances et valeurs d'autrui). Dans ce sens, la formation vise non seulement à rendre les apprenants plus autonomes dans leur capacité à agir comme professionnels, autonomes dans l'action d'un point de vue instrumental et situationnel, maitrisant les outils, les codes et les attentes du milieu auxquels ils sont préparés, mais aussi et surtout plus autonomes d'un point de vue épistémologique, c'est-à-dire capables d'interroger le bien-fondé de l'action, d'émettre des jugements informés et finalement d'influencer le milieu dans lequel ils agissent sur le plan des valeurs, de la déontologie, voire des idéologies (Eneau, 2005, 2011).

En résumé, il s'agit non seulement de prendre en compte la demande de montée en compétences, d'outillage ou de boites à outils que peuvent réclamer les apprenants, en particulier lorsqu'ils doivent apprendre à créer ou gérer des dispositifs complexes et répondre à des prescriptions, mais de ne surtout pas s'arrêter à cette demande instrumentale et de les amener vers une démarche plus réflexive et plus critique. La mise à distance de la certification notamment (pour décentrer les étudiants du seul objectif d'obtention du diplôme) s'avère ainsi nécessaire pour accorder la priorité non à la maitrise d'outils (gestion de projet, construction de référentiels, maîtrise financière, etc.) mais bien plus à la construction de capacités de distanciation. Il s'agit, alors, dans 
une perspective d'auto-formation transformatrice, d'accompagner des sujets autonomes se produisant (Mezirow, 2001).

\section{Méthodologie : une formation-action-recherche comme espace dialogique}

C'est, là, tout l'enjeu de la recherche-action que nous menons actuellement auprès de professionnels ou futurs professionnels de la formation, directement concernés par ces questions. A la croisée d'une méthodologie classique de recherche-action et d'une recherche menée en cours de formation, avec et pour les apprenants, nous avons choisi de qualifier cette démarche de "formation-action-recherche", signalant par l'ordre des termes choisis les priorités qui articulent ces trois temps imbriqués, encastrés l'un à l'autre'.

16 A l'Université Rennes 2, notre master accueille 20 à 25 apprenants par année (soit une cinquantaine de personnes, réparties entre master 1 et master 2), dont une majorité de professionnels qui reprennent leurs études pour valider leur expérience et obtenir un diplôme. Notre programme poursuit donc, d'une part, des visées instrumentales d'accès et de préservation de l'emploi, via l'acquisition de compétences sanctionnées par un diplôme, mais il vise aussi, d'autre part, l'accompagnement des sujets dans leur réalisation personnelle et leur développement en tant que sujets sociaux.

Dans ce programme de master, ces thématiques sont réfléchies pour accompagner la construction de la posture et de l'identité professionnelle, en offrant aux étudiants un espace dialogique de transformation personnelle, sociale et institutionnelle. En effet, si les pratiques d'enseignement à l'université n'échappent en rien aux logiques paradoxales décrites plus haut (logiques instrumentales d'acquisition de compétences et professionnalisation, d'acquisition de diplôme, etc.), elles offrent aussi, grâce à des temps longs de formation (une à deux années dans notre cas), la possibilité de mettre en œuvre une socio-pédagogie construite à partir de l'expérience réfléchie, une formation de soi par soi et de soi par autrui (Eneau, 2005).

La formation se déroule en alternance : les apprenants viennent une semaine par mois à l'université, de septembre à juin, et sont sur le terrain le reste du temps. Différentes unités de formation sont consacrées, lors des séances de regroupement, à l'analyse de pratiques et à la construction du projet professionnel. De plus, l'organisation pédagogique de l'alternance est outillée: un livret de professionnalisation (outil d'information partagée sur la démarche, les attendus, le rôle des différents acteurs, le calendrier global du parcours, les outils et la démarche d'évaluation) permet de poser les bases d'une démarche contractuelle à travers laquelle le projet de l'étudiant (et ses priorités en termes de professionnalisation), celui de l'université (avec son référentiel de formation) et celui de la structure d'accueil (en rapport avec son projet institutionnel ou bien conjoncturel) doivent converger.

Pour provoquer cette mise en dialogue, nous organisons en début d'année universitaire un temps collectif avec l'ensemble des tuteurs (environ $60 \%$ des tuteurs y participent), puis dans le cadre de rencontres entre le responsable de stage de l'université, le tuteur et l'étudiant, organisées autant que possible sur les sites professionnels. Les tuteurs participent également à la co-construction de la mission que réalisera l'étudiant en prenant part, lors des deux premiers mois de formation, à l'élaboration d'un cahier des 
charges. Basé sur le modèle de la pédagogie du contrat (contrat réciproque d'actions et d'apprentissages à la fois) et inspiré des outils utilisés par les professionnels du secteur, ce document permet de négocier les objectifs, les moyens et les livrables attendus en fin de mission.

Les tuteurs professionnels participent à cette forme d'alternance dialogique, rencontre problématique de différents mondes vécus, dans leurs différents intérêts, à la fois stratégiques, communicationnels et parfois émancipateurs. Les unités de formation en général, mais l'analyse de pratiques en particulier, sont ensuite les lieux d'exploration et d'explicitation de l'expérience vécue, utilisant la discussion comme espace de formation dialogique (Jacques, 2004 ; Labelle, 1996). Animés par l'universitaire qui pilote le dispositif de stage (enseignant-référent de l'équipe pédagogique), ces espacestemps de formation permettent d'aborder de manière réflexive non seulement l'expérience vécue sur les lieux professionnels, mais aussi l'expérience antérieure et les «cadres de références» des étudiants (Brookfield, 2005 ; Mezirow, 2001), dans toute leur singularité et leur diversité.

21 Le dispositif de formation s'inscrit ainsi dans une logique de formation à et par la recherche, par la réflexion dans l'action et sur l'action ; l'alternance est donc au cœur de la formation. Plus encore, nous considérons ce dispositif comme un lieu de confrontation, de disputatio, qui questionne les logiques de la formation, de l'action située et de la recherche à la fois, avec des visées heuristiques de production de savoirs généralisables, mais aussi de savoirs plus locaux, plus expérientiels. En effet, il se situe dans l'expérimentation et la mise en situation, pour les apprenants, tout autant qu'il est dirigé par l'observation et l'analyse des pratiques. Les conduites des étudiants pendant les travaux individuels et collectifs à l'université, la production des travaux réflexifs demandés dans le cadre de la formation, mais aussi la production individuelle de l'écrit de fin d'année (le mémoire qui valide le master, en deuxième année) sont autant de lieux et de moments où nous étudions le dialogisme expérientiel (Bertrand, 2007), c'est-à-dire la combinaison de logiques vécues, souvent de manière paradoxale, de maîtrise technique, de développement intellectuel, de réflexivité et d'intercompréhension.

Enfin, pour conduire cette recherche, nous sommes en position d'observation participante. L'observation des situations d'échanges, lors des regroupements à l'université, est une source d'information continue sur l'évolution des capacités réflexives des étudiants et sur les distorsions de sens vécues (Mezirow, 1991). Les productions individuelles des apprenants (dossiers écrits à l'issue des stages, mémoires de fin d'année) et les productions collectives (dossiers élaborés au cours des deux années) constituent les principales sources écrites de nos données. Elles nous permettent notamment d'étudier la manière dont les travaux de recherche sont issus d'une construction individuelle et collective, réflexive et transformative, à partir de la mise à distance de l'expérience vécue, énoncée et théorisée.

\section{Compte-rendu : quelques résultats de la formation- action-recherche}

Sans avoir de valeur prescriptive ou normative, les premiers constats de cette expérimentation, que nous présentons ici, permettent d'illustrer la manière dont la perspective transformatrice peut être mobilisée dans un tel dispositif de formation 
professionnalisante. Deux séries de résultats seront présentés: d'une part, l'organisation des unités de formation comme espaces de production individuelle d'une réflexion critique, d'autre part, les usages collectifs que peuvent faire les étudiants de cette réflexion dans leurs perspectives transformatrices. Deux exemples en particulier permettront d'illustrer ces résultats; sans vouloir réduire les liens entre les unités de formation et les processus transformateurs opérés (un seul module de formation n'entraînant pas une seule nature de transformation) ni prétendre à un quelconque idéal-type (dans les exemples donnés ci-après), ils offrent par contre une lecture plus concrète des modélisations proposées.

\subsection{Implications individuelles de la perspective transformatrice, en cours de formation}

L'unité de formation « Analyse des pratiques » vise l'apprentissage d'une démarche à la fois auto et co-réflexive sur l'activité de travail, portant notamment sur les questions d'éthique et de positionnement professionnel des apprenants. Les temps d'analyse de pratiques, à raison d'une demi-journée par mois sur cinq mois, constituent des moments où les conditions du dialogue rationnel et réflexif sont rassemblées (Mezirow, 2001). Ce sont des espaces et des temps propices, pour chacun, à l'exercice de l'intercompréhension qui découle d'un double mouvement de décentration (distinction des trois mondes: soi, les autres, l'environnement) et de réflexivité (comme questionnement des prétentions à valider des actes de langage, vécus en situation de travail et vécus en formation). Ce mouvement de transformation des apprenants, réalisé individuellement mais par l'apport des autres membres du groupe, peut être modélisé comme suit :

Fig. 1 : Modélisation du processus d'apprentissage transformateur dans l'analyse des pratiques

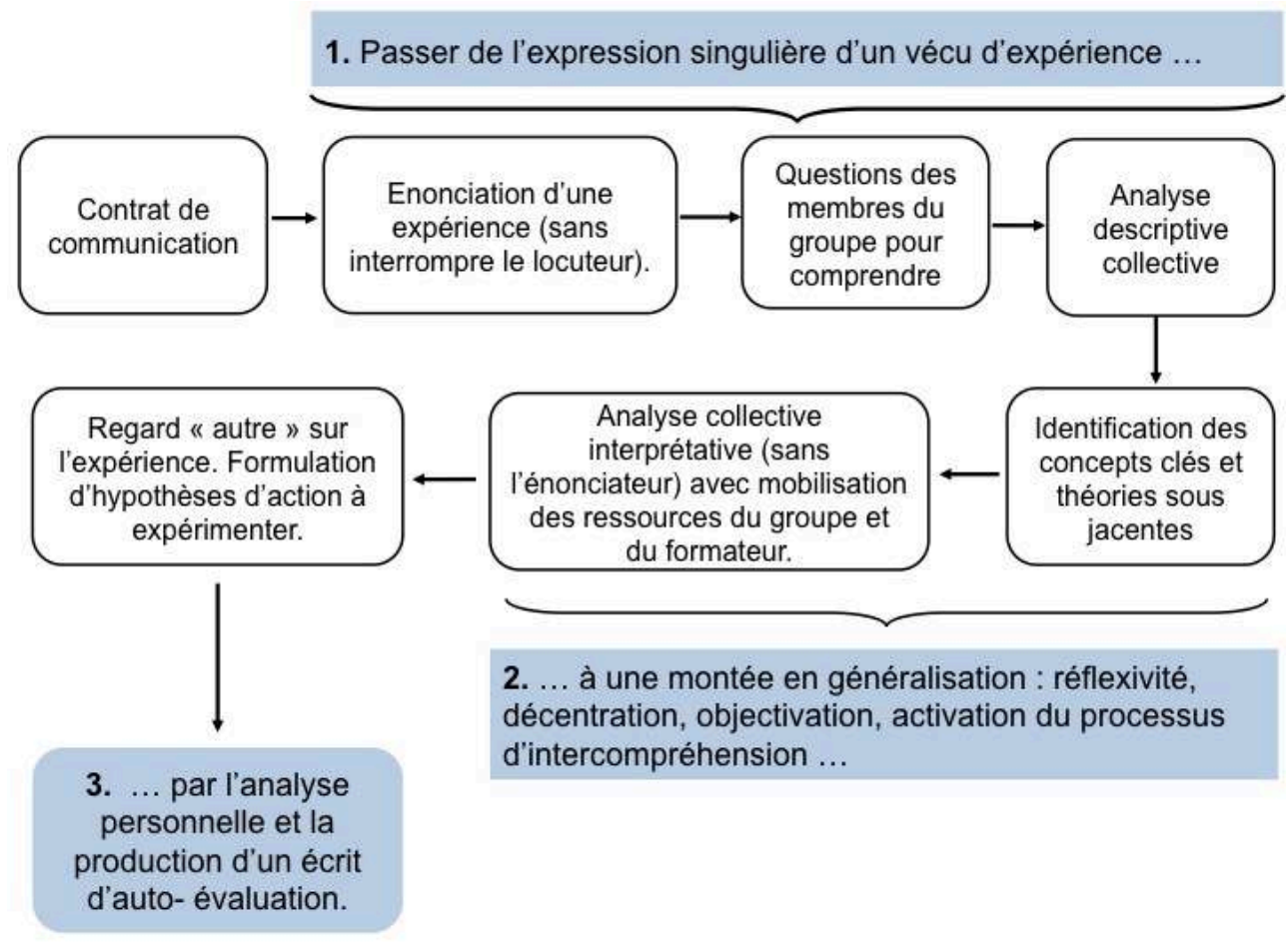


mouvement d'auto et de co-formation, comme processus progressif de décentration et de réflexivité par lequel la situation narrée va changer de statut pour devenir un objet de travail, est illustré par l'analyse d'une situation énoncée par A., étudiante de la promotion 2010-2011. d'offre dans l'organisme de formation où elle effectue son stage. Au fil du dialogue réflexif, pendant le module de formation, elle comprendra ce qu'elle n'avait pas formalisé clairement. Sa mission a la particularité d'être évaluative : réaliser une étude dans la perspective d'améliorer des pratiques de réponse aux appels d'offre suggère qu'elle s'appuie sur la description et l'analyse critique de ce que font les responsables de formation de la structure où elle effectue son stage. Elle parle de sa difficulté à organiser et à animer des groupes de travail, des tensions palpables au sein des équipes, de sa difficulté à prendre du recul en situation. Elle parle de la place ambiguë qu'on lui donne au sein de l'organisme, mais peine à parler de la place qu'elle veut prendre. Elle dit se sentir bloquée, avoir des difficultés dans sa prise de recul, parle de la confusion de ses intérêts (devenir ingénieur de formation et se professionnaliser, n'être que stagiaire mais vouloir s'intégrer à l'équipe, etc.) et évoque les enjeux d'embauche à la fin du master 2, au sein de cet organisme.

A partir d'un repérage des mots-clés tirés de l'activité de langage (tensions interpersonnelles, recul et implication, évaluation d'une pratique, travail d'équipe, place donnée, revendiquée ou espérée, etc.), le groupe s'entendra pour définir l'expérience de A. comme l'épreuve d'une construction de la posture professionnelle, difficile à tenir dans une activité d'évaluation. Les modèles théoriques mobilisés par les participants pour redéfinir la situation comme les réponses d'action proposées à $\mathrm{A}$. seront, quant à eux, généralisables; ils portent sur la construction identitaire professionnelle, les transactions à l'œuvre entre identité pour soi et identité pour autrui, les théories de la régulation conjointe (Raynaud, 1997), etc. Les hypothèses d'action à expérimenter, proposées par le groupe à la suite de l'analyse collective, sont les suivantes :

- Penser l'évaluation et l'amélioration des pratiques de réponses à appels d'offre dans son organisme selon la théorie de la régulation conjointe (entre régulation de contrôle, en appui sur le référentiel d'activités officiel pour répondre à un appel d'offre et régulation autonome, comme pratiques non formelles des acteurs, dans l'action);

- Créer les conditions de cette régulation conjointe (en termes d'instances, de modalités de travail, etc.), dans les groupes qu'elle aura à animer ;

- Revenir sur le cahier des charges de la mission de A. pour échanger avec le tuteur et l'enseignant référent du master sur la cohérence du projet de la structure et ce qu'elle attend, à titre personnel, en termes de professionnalisation et de projet professionnel.

Partant d'une problématique individuelle et valable pour un contexte singulier, le travail d'analyse réflexive menée par A., grâce au groupe de ses pairs, ressort ainsi d'une démarche à la fois auto-formatrice et co-formatrice. Le module d'analyse de pratiques permet à chacun de s'enrichir des apports des autres et de se former en transformant ses perspectives de sens. 


\subsection{Implications collectives de la perspective transformatrice, à l'issue de la formation}

L'unité de formation intitulée "Ingénierie des dispositifs de gestion des parcours professionnels » offre un autre exemple de dialogue réflexif, dans un cadre délibératif où l'apprentissage transformateur est exploré pour négocier et construire un objet d'étude. L'une des finalités attendues du travail, lorsqu'il est réinvesti dans le mémoire de fin d'études, est de proposer à la fois des grilles de lecture de situations professionnelles mais aussi des pistes d'action pour les professionnels (ou futurs professionnels) et surtout pour les collectifs investis. Les visées des apprentissages sont donc à la fois émancipatrices (apprendre à se connaître, apprendre à apprendre) mais aussi instrumentales et régulées par les formes d'un agir communicationnel, dans des dimensions individuelles et collectives. Ce dispositif peut être schématisé comme suit :

Fig. 2 : Modélisation du processus d'apprentissage transformateur construit à partir du modèle de la recherche-action (Barbier, 1996)
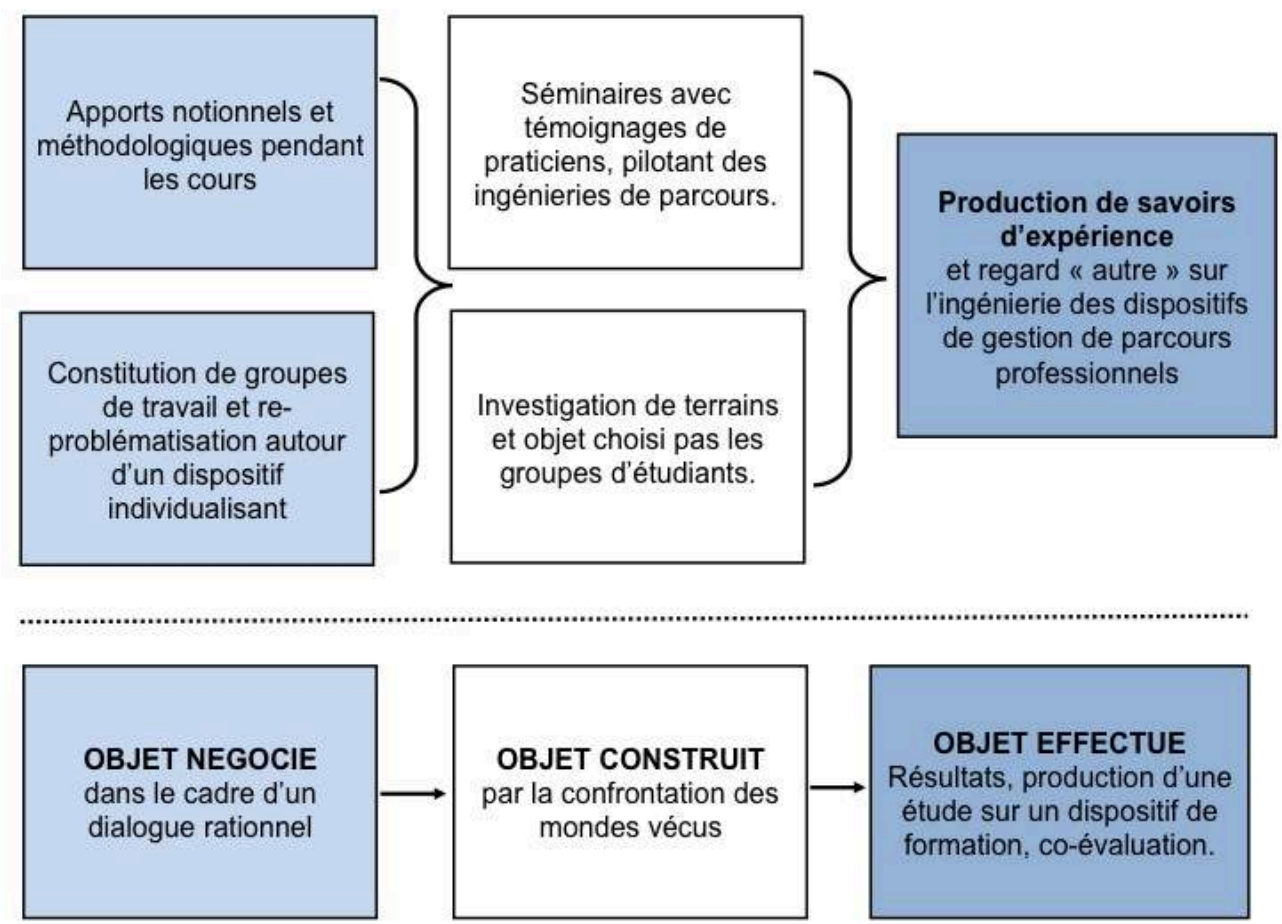

Le processus transformateur, dans son rapport avec le contexte professionnel des étudiants, du côté de l'entreprise et de l'action professionnelle, peut être illustré par la recherche menée par S. qui a progressivement élaboré son objet personnel de recherche, pendant son année de formation, l'a négocié avec l'équipe pédagogique, son directeur de mémoire, le groupe d'étudiants et ses collègues sur son terrain professionnel : elle l'a agi, réalisé, mis à l'épreuve.

31 Ancienne éducatrice au Ministère de la Justice, S. est responsable de formation, lorsqu'elle reprend ses études en 2009-2010. Elle a en charge l'élaboration et la mise en œuvre du plan de formation pour des éducateurs de la Protection Judiciaire de la Jeunesse. Sa recherche porte sur les évolutions du système de formation professionnelle de cette administration et en particulier sur l'analyse des besoins en 
formation des acteurs afin d'élaborer des réponses efficaces, justes et utiles. Identifiant les flux de communication et de décision, S. montre, à l'issue de son travail, la manière dont les pratiques sont mises en tension, notamment par la rencontre problématique d'une logique institutionnelle descendante qui vise la rationalisation du travail et de ses moyens (modèle tayloriste et production de formations adaptatives) avec des logiques d'acteurs issues de la culture du métier (plus ascendantes et plus délibératives).

Grâce à la mobilisation de la théorie de l'agir communicationnel d'Habermas, S. met au jour la rencontre problématique des différents mondes sociaux de son institution et l'absence d'une logique communicationnelle permettant de s'entendre sur la réalisation d'une action commune (le plan de formation). Elle en viendra à définir l'analyse des besoins de formation comme une action stratégique qui permet la rencontre du projet institutionnel avec celui des acteurs et le nécessaire travail d'élucidation et de négociation dans l'analyse des besoins de formation. En montrant l'écart grandissant entre travail prescrit et travail réel des éducateurs, sa recherche dévoilera une autre tension vécue par les acteurs: une souffrance grandissante au travail, de la part des éducateurs, que selon S., la formation devrait prendre en considération, tant dans ses dimensions politiques que fonctionnelles et opérationnelles.

Ici, la recherche constitue un objet potentiellement transformateur des savoirs, des pratiques et des représentations pour l'apprenante, mais elle constitue aussi une ressource, la production de savoirs singuliers, locaux, sur l'exercice d'un métier et ses problématiques de formation continue. Elle accompagne non seulement le développement d'un sujet, mais aussi la transformation, après coup, des acteurs et des organisations dans lesquels elle s'effectue.

\section{Ouverture : perspective transformatrice et gestion de la diversité}

34 La perspective transformatrice empruntée aux travaux de Mezirow montre ainsi, dans ses formes d'emploi possibles, deux exemples de mise à distance critique, par les apprenants, de leur expérience personnelle et professionnelle, de manière individuelle et collective, dans le groupe de formation (pendant les séquences de regroupement à l'université) mais aussi dans le milieu professionnel (pendant et à l'issue de la formation).

35 La transformation individuelle, pendant les séquences de formation à l'université, est un processus nécessairement long, qui s'élabore par étape, tout au long de l'année, à partir d'une perspective dialogique visant à interroger la manière dont l'expérience singulière peut être distanciée par la mise à l'épreuve de schèmes de sens autres que ceux des représentations individuelles de l'apprenant. L'énoncé de la situation fait ainsi parler, dans le cadre d'un dialogue rationnel, les mondes sociaux du travail et les mondes vécus par les participants au dialogue. Il mobilise des savoirs objectifs (des théories générales), des savoirs sociaux (règles, valeurs, représentations, croyances) et des savoirs subjectifs (tirés de l'épreuve des situations vécues par les sujets). Il interroge les distorsions de sens des participants qui sont à la fois d'ordre épistémique (savoirs non intégrés ou mal utilisés), sociolinguistique (usage plus ou moins correct d'un langage professionnel, parfois très technique) mais aussi psychologique (émotions 
et pensées parfois trompeuses). L'analyse est élaborée à partir d'un double processus d'auto-formation et de co-formation, conjointement construit dans un objet partagé, préservant cependant la singularité de l'action et de son vécu.

La transformation collective, quant à elle, peut être envisagée à deux niveaux : dans le groupe d'apprenants, tout au long des différents regroupements qui ponctuent l'année universitaire, par les processus de co-formation mis en œuvre, mais aussi dans les collectifs de travail investis par les apprenants pendant leur période de professionnalisation, sur le terrain. Selon nos observations, ce cas reste toutefois plus rare et semble se produire plus fréquemment chez les apprenants professionnels en reprise d'études que chez les plus jeunes ou les novices. De par leur expérience antérieure, les professionnels ont peut-être une vision plus globale et plus complexe du monde social dans lequel ils sont ancrés et arrivent aussi à l'université avec des interrogations à la fois d'ordre pratique et éthique. Malgré le peu de recul dont nous disposons, il nous semble ainsi plus fréquent de retrouver la posture de S. chez des apprenants qui investissent le temps de leur reprise d'études universitaires comme des moments de ré-interrogation profonde de leur identité professionnelle, voire de la confrontation de leurs valeurs avec celles de leur milieu professionnel d'origine.

De plus, tous les apprenants n'ont pas le temps, loin s'en faut, les capacités ou les marges de manœuvre suffisantes pour interroger et faire évoluer les pratiques du monde professionnel dans lequel ils ont, durant l'année ou les deux années de leur formation, à intervenir. Ici, se trouve ainsi l'une des principales limites du modèle mis en œuvre. Cependant, pour les apprenants en voie de professionnalisation comme pour les professionnels retournant aux études, la perspective transformatrice mobilisée montre, à différents niveaux, la manière dont elle peut contribuer à articuler, dans un dispositif reposant sur l'alternance, expérience singulière et expérience collective d'apprentissage, auto-formation et co-formation, transformation individuelle et collective, recompositions existentielles et identitaires.

Pour les plus jeunes des apprenants, qui ne sont pas encore complètement sortis du milieu universitaire et du monde de la formation initiale, la distance critique vis-à-vis des pratiques du milieu peine bien sûr parfois à dépasser les logiques instrumentales exigées par le discours managérial ou les contraintes imposées par la hiérarchie. A l'inverse, les prises de conscience réflexives nous sont plus fréquemment repérables chez les étudiants plus expérimentés (professionnels en reprise d'études), avec une appétence affirmée pour un processus transformateur recherché. Bien sûr, il semblerait vain de distinguer, entre ces deux publics, ceux qui, parmi les plus jeunes ou parmi les plus expérimentés, entrent dans une démarche transformatrice plus individuelle ou plus collective, plus centrée sur eux-mêmes ou plus centrée sur le milieu de travail, comme lieu d'investissement transformateur. Au contraire, s'il est bien un consensus sur lequel s'entendent les apprenants, c'est bien la richesse, selon leurs propres termes, qui émane d'une promotion constituée d'un groupe hétérogène, aux origines et aux expériences personnelles et professionnelles diversifiées.

39 C'est alors de la complexité de gérer cette diversité que ressort toute la difficulté, pour l'équipe pédagogique, dans un accompagnement à la fois personnalisé et exigeant pour chacun des apprenants, mais différencié et prenant en compte l'expérience antérieure, l'expérience vécue pendant l'année de formation, l'expérience réinvestie dans le collectif de travail, voire dans le monde social de chacun. En suivant le devenir des apprenants à l'issue de leur formation, des recherches futures pourraient nous 
permettre d'observer la manière dont la perspective transformatrice pourrait influencer le devenir de ces acteurs. Si cette perspective a réellement des implications existentielles, sociales, professionnelles et culturelles à la fois, comme le revendique Mezirow, il reste alors à analyser les éventuelles transformations organisationnelles que ces apprenants pourront réinvestir, dans les processus et les finalités même du monde du travail dans lesquels, ultérieurement, ils auront à agir.

\section{BIBLIOGRAPHIE}

Barbier, R. (1996). La recherche action. Paris : Anthropos.

Bertrand, E. (2007).L'expérience formatrice à La Poste : Vers une dialogique entre autoformation émancipatrice et coformation instrumentale. Thèse de doctorat non publiée en Sciences de l'Education. Université Paris 8.

Boltanski, L., \& Chiapello, E. (1999). Le nouvel esprit du capitalisme. Paris : Gallimard.

Brémaud, L., \& Guillamin, C. (dir.) (2010). L'archipel de l'ingénierie de la formation. Rennes : Presses universitaires de Rennes.

Brookfield, S. D. (2005). The Power of critical theory. Liberating adult Learning and teaching. San Francisco : Jossey-Bass.

Charlier, E. (2001). Former des enseignants-professionnels pour une formation continuée articulée à la pratique. Dans L. Paquay, M. Altet, E. Charlier \& P. Perrenourd (dir.), Former des enseignants professionnels (p. 97-117). Bruxelles : De Boeck.

Charlier, B. (2005). Parcours de recherche-action-formation. Revue des sciences de l'éducation, 31(2), 259-272.

De Lescure, E., \& Frétigné, C. (2010). Les métiers de la formation. Approches sociologiques. Rennes : Presses universitaires de Rennes.

Dejours, C. (2010). Observations cliniques en psychopathologie du travail. Paris : Presses universitaires de France.

Donaldson, J. F. (2009). Fostering transformative learning in leadership development. Dans J. Mezirow \& E.W. Taylor, W. (dir.), Transformative learning in practice. Insights from community, workplace and higher education. (p. 67-77). San Francisco, CA : Jossey-Bass.

Eneau, J. (2005). La part d'autrui dans la formation de soi. Autonomie, autoformation et réciprocité en contexte organisationnel. Paris : L'Harmattan.

Eneau, J. (2008). From reciprocity to autonomy, or vice-versa? The contribution of french personalism to a new perspective of self-directed learning. Adult Education Quarterly, 58(3), 229-248.

Eneau, J. (2011). Développement de l'autonomie au travail : les apports de la réciprocité à une modèle de l'autoformation en contexte organisationnel. DansL. Jacquot (dir.), Travail et dons (p. 215-234). Nancy : Presses universitaires de Nancy. 
Freire, P. (2006). Pédagogie de l'autonomie. Savoirs nécessaires à la pratique éducative. Toulouse : Eres.

Habermas, J. (1997). Théorie de l'agir communicationnel. T2 : Pour une critique de la raison fonctionnaliste. Paris : Fayard.

Habermas, J. (2001). Théorie de l'agir communicationnel. T1 : Rationalité de l'agir et rationalisation de la société. Paris : Fayard.

Honneth, A. (2008). La société du mépris. Vers une nouvelle théorie critique. Paris : La Découverte.

Jacques, F. (dir.) (2004). Education et formation. Essai sur quelques constantes éducatives. Paris : Parole et Silence.

Labelle, J.-M. (1996). La réciprocité éducative. Paris : Presses universitaires de France.

Lameul, G. (2009). Penser ensemble et de façon solidaire sujet et situation au sein des dispositifs. Dans G. Lameul, A. Jézégou \& A.-F. Trollat (dir), Articuler dispositifs de formation et dispositions de l' apprenant (p. 21-44). Lyon: Chronique sociale.

Leclerq, E., \& Niclot, D. (dir.). (2010). Former des professionnels de la formation en Europe. Reims : Presses universitaires de Reims.

Mezirow, J. (1991). Transformative dimensions of adult learning. San Francisco : Jossey-Bass.

Mezirow, J. (2000). Learning to think like an adult. Core concepts of transformation theory. Dans J. Mezirow (dir.), Learning as transformation. Critical perspectives on a theory in progress (p. 3-33). San Francisco : Jossey-Bass.

Mezirow, J. (2001). Penser son expérience. Une voie vers l'autoformation. Lyon : Chronique Sociale.

Mezirow, J. (dir.). (2000). Learning as transformation. Critical perspectives on a theory in progress. San Francisco : Jossey-Bass.

Mezirow, J., \& Taylor, E. W. (2009). Transformative learning in practice. Insights from community, workplace and higher education. San Francisco : Jossey-Bass.

Pineau, G. (2010). Concevoir, construire, conduire de la formation, avec génie, ingéniosité et générosité. Dans L. Brémaud \& C. Guillaumin (dir.), L'archipel de l'ingénierie de la formation (p. 9-13). Rennes : Presses universitaires de Rennes.

Raynaud, J.-D. (1997). Les Règles du jeu : L'action collective et la régulation sociale. Paris : Armand Colin.

Tremblay, N., \& Eneau, J. (2006). Sujet, société et autoformation - regards croisés du Québec et de France. Education Permanente, 168, 75-88.

Sorel, M., \& Wittorski, R. (dir.). (2010). La professionnalisation en actes et en questions. Paris :

L'Harmattan

\section{NOTES}

1. Le master dont il est ici question s'intitule "Stratégie et Ingénierie en Formation d'Adultes » (SIFA). Il se déroule sur deux années (Master 1 et Master 2), à l'Université de Rennes 2. Héritier d'un diplôme existant depuis la fin des années 1980, il prépare, au niveau Bac+5 années d'études supérieures, de futurs responsables de formation (responsables en entreprise ou en organismes de formation, ingénieurs de formation, consultants, etc.). 
2. Nous nous inspirons ici de la démarche classique de « recherche-action » (Barbier, 1996) et de la démarche de "recherche-action-formation » (Charlier, 2001 ; Charlier, 2005), tout en s'en distinguant par l'ordre des termes.

\section{RÉSUMÉS}

Parmi les défis posés, aujourd'hui, à l'université par l'alternance et la professionnalisation, il s'agit d'imaginer des articulations entre savoirs pratiques issus du monde du travail et savoirs théoriques issus du monde de la recherche, dans des dispositifs qui permettent de concilier des tensions paradoxales. Illustrant ces tensions, notre contribution vise à décrire l'ingénierie pédagogique mise en œuvre dans un dispositif de professionnalisation aux métiers de la formation. L'utilisation des expériences, grâce à l'alternance, comporte pour les apprenants différents enjeux : construire à partir de l'action une réflexion sur sa posture professionnelle, se construire une identité à la fois personnelle et collective, construire une réflexion éthique basée sur la distanciation et la mobilisation de savoirs critiques. La mise à distance de la diplômation s'avère alors nécessaire pour accorder la priorité non à la maîtrise d'outils mais à la construction de capacités réflexives, dans une perspective d'autoformation transformatrice.

\section{INDEX}

Mots-clés : alternance, auto-formation, co-formation, perspective critique, professionnalisation, réflexivité

\section{AUTEURS}

\section{JÉRÔME ENEAU}

Département des sciences de l'éducation, Université de Rennes 2, France

Place du recteur Henri Le Moal, CS 24307, F-35043 Rennes Cedex.

jerome.eneau@univ-rennes2.fr

\section{ERIC BERTRAND}

Département des sciences de l'éducation, Université de Rennes 2, France

Place du recteur Henri Le Moal, CS 24307, F-35043 Rennes Cedex.

\section{GENEVIÈVE LAMEUL}

Département des sciences de l'éducation, Université de Rennes 2, France 
Place du recteur Henri Le Moal, CS 24307,

F-35043 Rennes Cedex. 